\title{
Infestación por Cladocolea loniceroidesy sus potenciales aves dispersoras de semillas en un área verde urbana de la ciudad de Aguascalientes, México
}

\section{Cladocolea loniceroides infestation and its potential seed-dispersing birds in an urban green area of Aguascalientes city, Mexico}

\author{
Ivoon Alejandra Martínez-Castruita', Manuel Higinio Sandoval-Ortega², Moisés Arellano-Delgado' y Victor Manuel \\ Martínez-Calderón ${ }^{3}$
}

\begin{abstract}
1 Universidad Autónoma de Aguascalientes. Departamento de Biología. Centro de Ciencias Básicas. Aguascalientes, Aguascalientes, México.
\end{abstract}

2 Universidad Autónoma de Aguascalientes. Departamento de Biología. Centro de Ciencias Básicas. Herbario HUAA. Aguascalientes, Aguascalientes, México.

\author{
3 Universidad Autónoma de Aguascalientes. Depar- \\ tamento de Ciencias Agronómicas. Centro de Ciencias \\ Agropecuarias. Aguascalientes, Aguasca-lientes, \\ México. \\ * Autor de correspondencia. m.higinio.s@hotmail.com
}

\section{RESUMEN}

Los muérdagos verdaderos pertenecen al orden Santalales, que está integrado por 13 familias, entre las que se encuentra Loranthaceae. Muchas de estas plantas hemiparásitas dependen de las aves para la dispersión de sus semillas. Son considerados como una plaga forestal, al grado de que en México se consideran como el segundo agente de destrucción de los bosques. El objetivo del presente trabajo fue determinar las especies arbustivas y arbóreas afectadas por el muérdago verdadero Cladocolea loniceroides dentro del Parque Rodolfo Landeros Gallegos, la mayor área verde pública de la ciudad de Aguascalientes, evaluar el grado de infestación de las plantas hospederas e identificar las potenciales aves dispersoras de este muérdago en el sitio. En el área de estudio hay 2343 individuos infestados, pertenecientes a 16 especies de nueve familias. De la población total de las especies hospederas, $56 \%$ presentan infestación. Se identificaron cinco especies de aves, pertenecientes a cuatro familias, como potenciales dispersores de semillas de C. loniceroides. Debido a la proporción de individuos infestados, se considera grave el estado fitosanitario de la zona de estudio. Se recomienda sustituir las especies susceptibles a infestación por especies nativas no hospederas de C. loniceroides.

PAlabras Clave: muérdago, aves, ornitocoria, Pitangus sulphuratus, Pyrocephalus rubinus, Tyrannus vociferans.

\section{ABSTRACT}

True mistletoes belong to the order Santalales, which is made up of 13 families, among which is Loranthaceae. Many of these hemiparasitic plants depend on birds as seed dispersers and are considered a forest pest. In Mexico, they are the second agent of forest destruction. The objective of this work is to determine the shrub and tree species affected by Cladocolea loniceroides within the Rodolfo Landeros Gallegos Park, the largest public green area in the city of Aguascalientes, to assess the degree of infestation and to identify the main potential seed disperser birds of this mistletoe on site. In the study area there are 2343 infested individuals, belonging to 16 species from nine families. $56 \%$ of the total population of the host species present infestation. Five potential seed-dispersing bird species belonging to four families were identified. Due to the proportion of infested individuals, the phytosanitary status of the study area is considered serious. It is recommended to replace the species susceptible to infestation with native non-host species of $C$. loniceroides.

KEYWORDs: mistletoe, birds, ornithochory, Pitangus sulphuratus, Pyrocephalus rubinus, Tyrannus vociferans. 


\section{INTRODUCCIÓN}

Las plantas parásitas se distribuyen globalmente y son un componente integral en la mayoría de los ecosistemas (Mathiasen, Nickrent, Shaw y Watson, 2008), dentro de este grupo de plantas se encuentran los muérdagos, que se distribuyen en áreas forestales tanto tropicales como templadas, zonas áridas e incluso en áreas verdes urbanas de todos los continentes (Aukema y Martinez-del Rio, 2002; De la Paz Pérez-Olvera, Ceja-Romero y Vela-Ramírez, 2006; Mathiasen et al., 2008; Start, 2011).

Los muérdagos son plantas hemiparásitas aéreas, pertenecientes al orden Santalales (Judd, Campell, Kellogg, Stevens y Donoghue, 2016; Mathiasen et al., 2008; Stevens, 2001), que utilizan a su hospedero para extraer agua y nutrientes, impiden su desarrollo e incluso pueden causar su muerte (Alvarado-Rosales y Saavedra-Romero, 2005; Arriola Padilla, Velasco Bautista, Hernández Tejeda, González Hernández y Romero Sánchez, 2013). Suelen ser separados en dos grupos: enanos y verdaderos (Geils, Cibrián-Tovar y Moody, 2002; Vázquez Collazo, Villa Rodríguez y Madrigal Huendo, 2006).

Los muérdagos enanos incluyen únicamente especies del género Arceuthobium M. Bieb. (Geils et al., 2002), que pertenece a la familia Santalaceae (Stevens, 2001). Este grupo se distingue de los muérdagos verdaderos debido a su menor tamaño, sus individuos presentan hojas reducidas a escamas, parasitan exclusivamente coníferas y, por lo común, dispersan sus semillas de manera autónoma, pues las disparan, por medio de sus frutos explosivos, a distancias de hasta 10 m (Geils et al., 2002; Mathiasen et al., 2008; Vázquez Collazo et al., 2006). Aunque también se distribuyen en el Viejo Mundo, la mayor diversidad de muérdagos enanos se encuentra al noroeste de México y oeste de Estados Unidos (Geils et al., 2002; Mathiasen et al., 2008).

Por otro lado, se les conoce como muérdagos verdaderos al resto de especies de este tipo de plantas hemiparásitas. En este grupo se pueden encontrar representantes de las familias Loranthaceae, Misodendraceae y Santalaceae; sus hospederos pueden ser tanto gimnospermas como angiospermas arbustivas y arbóreas, la mayoría tienen hojas bien desarrolladas y dependen principalmente de las aves como dispersores de sus semillas, excepto por los géneros Nuytsia R. Br. ex G. Don (Lorantaceae) y Misodendrum Banks ex DC. (Misodendraceae), que se dispersan por el viento (Geils et al., 2002; Mathiasen et al., 2008; Stevens, 2001; TerceroBucardo y Rovere, 2010; Vázquez Collazo et al., 2006).

En México, el muérdago es la segunda plaga forestal con mayor promedio de superficie afectada a nivel nacional y el segundo agente de destrucción de los bosques después de los escarabajos descortezadores (Geils et al., 2002; Secretaría de Medio Ambiente y Recursos Naturales [Semarnat], 2019). En la actualidad se tiene registro de nueve géneros y alrededor de 150 especies de muérdagos distribuidos en el territorio nacional, que corresponden a las familias Loranthaceae y Santalaceae, de los cuales ocho géneros y 131 especies son muérdagos verdaderos (Vázquez Collazo et al., 2006; Villaseñor, 2016). Los géneros de muérdagos verdaderos distribuidos en el país son Antidaphne Poepp. \& Endl., Dendropbthora Eichler y Phoradendron Nutt, pertenecientes a Santalaceae; mientras que Cladocolea Tiegh, Oryctanthus (Griseb.) Eichler, Phthirusa Mart., Psittacanthus Mart. y Struthanthus Mart., pertenecen a Loranthaceae (Geils et al., 2002; Stevens, 2001; Kuijt, 2011; Vázquez Collazo et al., 2006; Villaseñor, 2016).

El género Cladocolea es nativo del nuevo mundo e incluye alrededor de 25 especies (Calderón de Rzedowski, 2001; Kuijt, 1975; Villaseñor, 2016); la mayoría de las cuales se concentra al norte del istmo de Tehuantepec y unas pocas se distribuyen en Centroamérica y Sudamérica (Kuijt, 1975). Del total de especies, 19 son nativas de México y 17 son endémicas (Villaseñor, 2016).

Cladocolea loniceroides (Van Tieghem) Kuijt se ha identificado como parásita de un amplio número de especies de diversas familias, en comparación de otras especies del mismo género (Alvarado-Rosales y SaavedraRomero, 2005), afectando zonas forestales y áreas verdes dentro de la República Mexicana (Alvarado-Rosales y Saavedra-Romero, 2005; Calderón de Rzedowski, 2001; Cid-Villamil y Bye, 1998; De la Paz Pérez-Olvera et al., 2006; Sandoval-Ortega y Siqueiros-Delgado, 2019). Como 
la mayoría de los otros muérdagos verdaderos, C. loniceroides depende principalmente de las aves para dispersar sus semillas y sus frutos muestran una variedad de atributos relacionados con la ornitocoria, pues son grandes, dulces, de color llamativo cuando maduros, con pedúnculos pequeños o nacidos directamente en la rama (Cid-Villamil, 2006; Mathiasen et al., 2008; Watson, 2001). En el estado de Aguascalientes, $C$. loniceroides es el único representante del género y hasta el momento se ha localizado solamente en el parque Rodolfo Landeros Gallegos (PRLG), dentro de la ciudad de Aguascalientes (Sandoval-Ortega y SiqueirosDelgado, 2019). Se ha observado que dentro de esta área verde urbana existe un gran número de árboles y arbustos infestados por esta especie de muérdago y debido a la zona en la que se encuentra, así como a su forma de dispersión natural, representa una amenaza para áreas verdes adyacentes y la vegetación nativa de Aguascalientes.

\section{OBJETIVOS}

El objetivo del presente trabajo fue determinar el grado de infestación de $C$. loniceroides en el PRLG, identificar las especies de árboles y arbustos afectadas y las principales especies de aves potencialmente dispersoras.

\section{MATERIALES Y MÉTODOS}

\section{Área de estudio}

El PRLG se encuentra ubicado dentro de la ciudad de Aguascalientes, en el municipio de Aguascalientes, a una altura de $1880 \mathrm{~m}$ s.n.m. Cuenta con una superficie de 88 hectáreas, 70 de las cuales corresponden a áreas verdes y el resto a construcciones y cuerpos de agua artificiales (Fig. 1). La vegetación del sitio está integrada principalmente por especies ornamentales introducidas y algunos elementos nativos de vegetación xerófila y bosque de galería. El clima es semiseco templado con lluvias de verano (BS1 kw $[\mathrm{w}])$, con temperatura media anual de $14{ }^{\circ} \mathrm{C}$ a $18{ }^{\circ} \mathrm{C}$ y precipitación total de $400 \mathrm{~mm}$ a $700 \mathrm{~mm}$ al año (Secretaría del Medio Ambiente y Desarrollo Sustentable [Semadesu], 2013).

\section{Identificación de especies hospederas de} Cladocolea loniceroides

El presente estudio se llevó a cabo de diciembre de 2018 a septiembre de 2019. Se inició con un muestreo exploratorio para identificar las especies de árboles y arbustos que presentan infestación por muérdago y aquellas especies no afectadas. Debido a que la mayoría de especies arbóreas y arbustivas presentes en el área de estudio son ornamentales, la identificación fue realizada en campo por medio de manuales (De la Cerda-Lemus y González-Adame, 2009; Semadesu, 2013). Para aquellas especies que no pudieron ser identificadas en campo se colectó una muestra utilizando la metodología propuesta por Engelmann (1986) y se transportaron al Herbario de la Universidad Autónoma de Aguascalientes (HUAA), donde se identificaron utilizando bibliografía especializada (De la Cerda-Lemus, 1989, 2000; Siqueiros-Delgado, 1996) y se cotejaron con ejemplares depositados en el mismo.

\section{Determinación del grado de infestación por Cladocolea loniceroides}

Debido a que el área de estudio corresponde a un área verde urbana, y las especies de interés no presentan una distribución aleatoria, se optó por cuantificar uno a uno la totalidad de individuos afectados. Los valores de infestación de cada uno de los árboles y arbustos contabilizados fueron registrados en una libreta de campo y cada individuo fue marcado para evitar errores de conteo.

Para determinar el grado de infestación se aplicó el sistema de seis clases propuesto por Hawksworth (1977), con algunas modificaciones (Fig. 2). La copa de árboles y arbustos fue dividida en tercios. A cada uno de estos se le asignó un valor dependiendo del número de matas de muérdago presentes, en el caso de arbustos y árboles menores a $3 \mathrm{~m}$ de altura, o de la proporción de ramas infestadas, en el caso de árboles con altura mayor a $3 \mathrm{~m}$ (Tabla 1). Los valores de cada uno de los tercios fueron sumados para obtener el valor total de infestación, el cual fue clasificado en uno de cuatro niveles (Tabla 2). El grado de infestación fue determinado únicamente para aquellas especies en las que se observó al menos un individuo con alguna mata de muérdago. 
Una vez cuantificada la totalidad de árboles infestados, se calculó para cada especie el valor de infestación promedio, incidencia de infestación (porcentaje de individuos afectados), incidencia de infestación ligera (porcentaje de individuos con infestación ligera), incidencia de infestación severa (porcentaje de individuos con infestación severa) y la densidad (número de individuos infestados por hectárea). Se consideró la superficie total de áreas verdes en la zona de estudio:

$$
\text { Densidad }=\frac{N}{\text { área }}
$$

$N=$ Individuos infestados

área $=70$ hectáreas

\section{Identificación de aves potencialmente dispersoras} de semillas de Cladocolea loniceroides

Fueron seleccionadas tres de las zonas con mayor número de árboles infestados; en ellas se estableció un sitio, sin diámetro definido, para observar las aves que se alimentaban de frutos de muérdago (Fig. 1). De diciembre 2018 a abril 2019, meses en los que $C$. loniceroides se encuentra en fruto, los lunes, cuando el PRLG permanece cerrado para el público y la presencia humana es menor que durante el resto de la semana, se visitó uno de los tres sitios establecidos para llevar a cabo el registro de aves con ayuda de binoculares y cámara fotográfica, de 7:00 a 12:00 del día. Se realizaron en total 18 muestreos, que equivalen a 90 horas de observación.

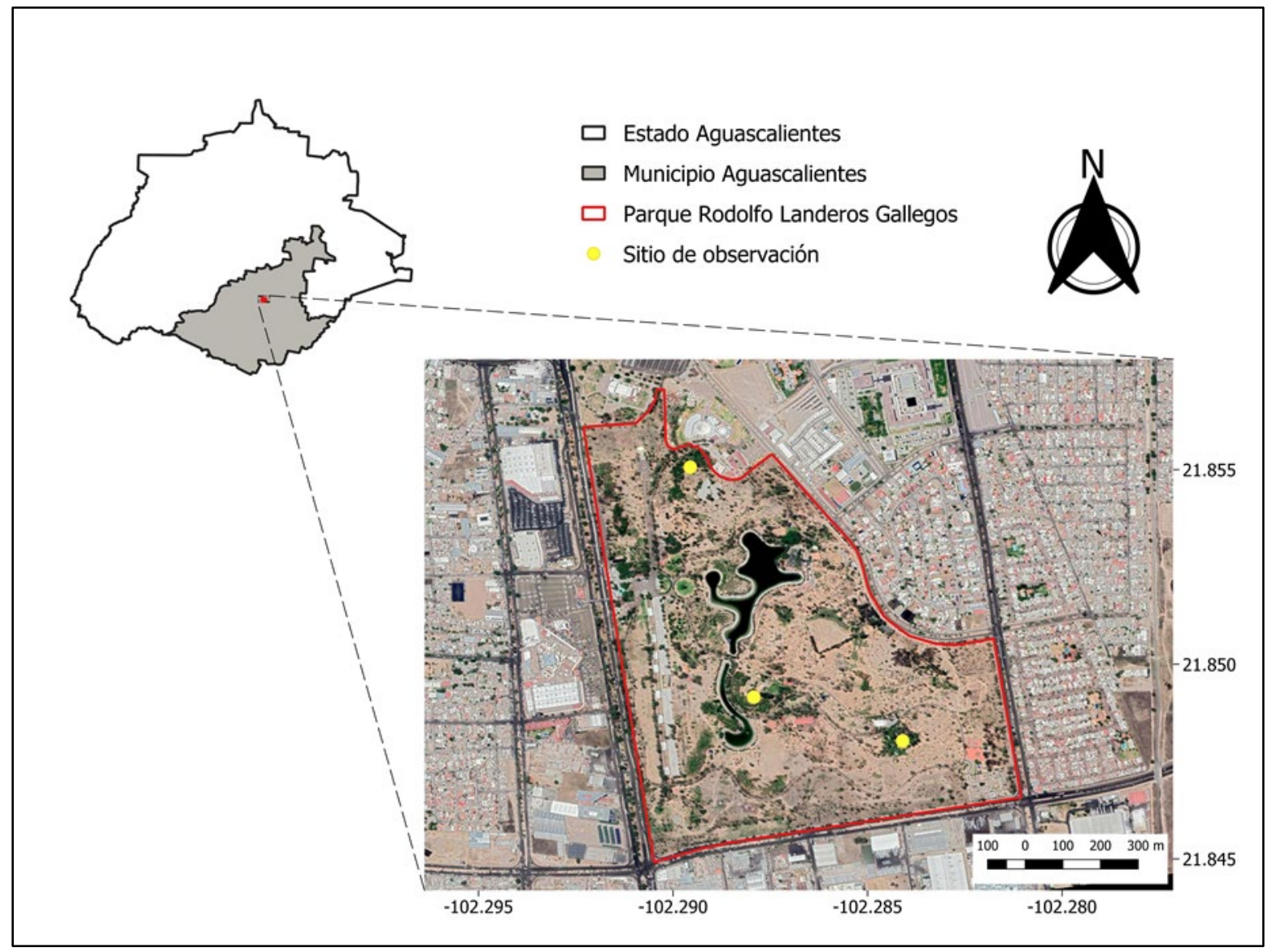

FiguRA 1. Ubicación del área de estudio y sitios de observación de aves, dentro del parque Rodolfo Landeros Gallegos, Aguascalientes, México. 


\section{A}

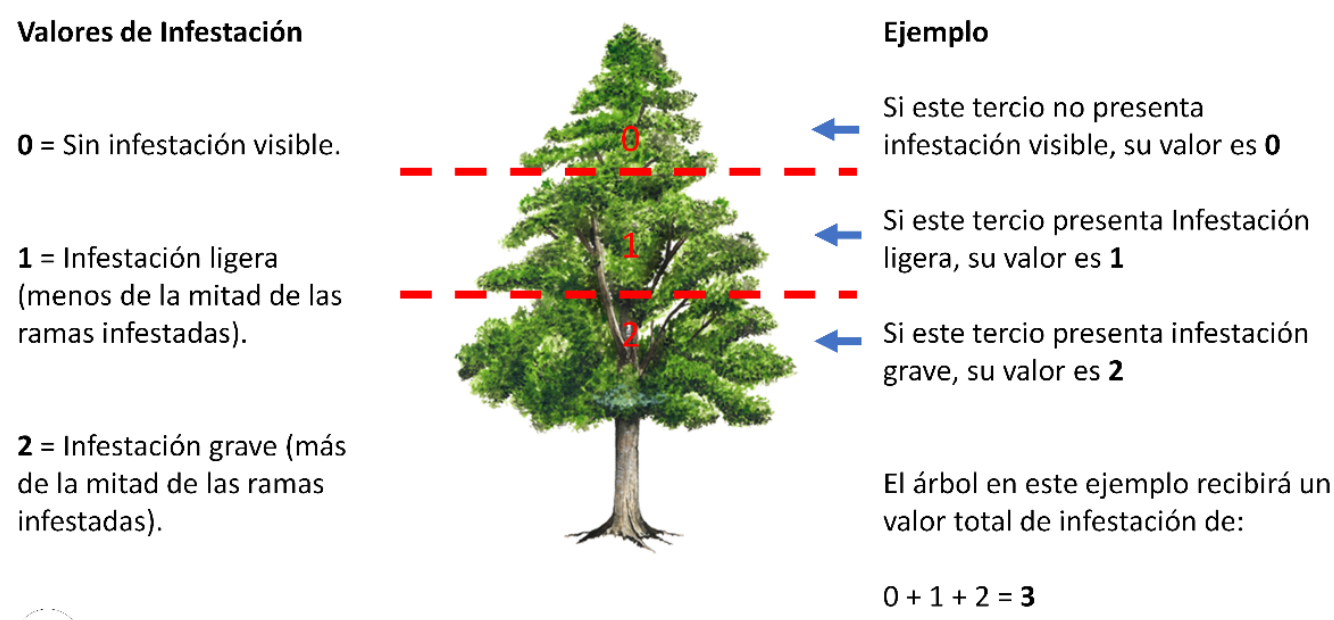

B

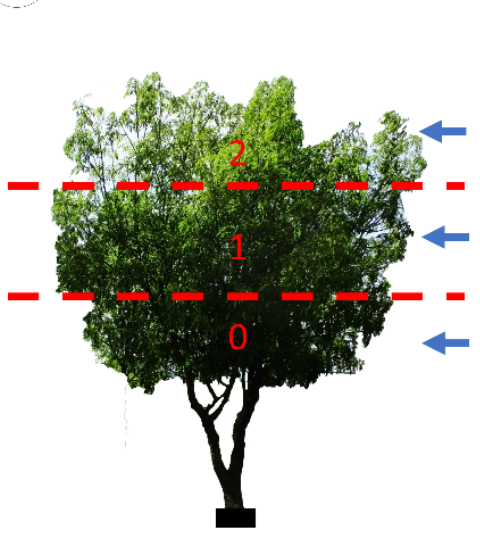

Ejemplo: árbol $>3 \mathrm{~m}$ de altura

Ejemplo: arbusto o árbol $<3 \mathrm{~m}$ de altura.

Si este tercio presenta más de la mitad de las ramas infestadas, su valor es: $\mathbf{2}$

Si este tercio presenta menos de la

mitad de las ramas infestadas, su valor es: 1

Si este tercio no presenta

infestación visible, su valor es: $\mathbf{0}$
Si este tercio presenta 3 o más matas de muérdago, su valor es: 2

Si este tercio presenta de 1 a 2

matas de muérdago, su valor es: 1

Si este tercio presenta matas de muérdago, su valor es: $\mathbf{0}$

En ambos ejemplos se obtiene un valor total de infestación de:

$$
2+1+0=3
$$

FIGURA 2. Valores de infestación, por muérdago, de árboles dentro del parque Rodolfo Landeros Gallegos (PRLG), Aguascalientes, México. A: sistema de seis clases de Hawksworth (1977). B: modificaciones realizadas al sistema de Hawksworth (1977) en el presente estudio.

TABLA 1. Valores asignados a la infestación por muérdago Cladocolea loniceroides en árboles y arbustos, dentro del parque Rodolfo Landeros Gallegos, Aguascalientes, México.

\begin{tabular}{lll}
\hline Valor & Árbol $>3 \mathrm{~m}$ & Arbusto o árbol $<3 \mathrm{~m}$ \\
\hline 0 & $\begin{array}{l}\text { Sin infestación } \\
\text { visible }\end{array}$ & $\begin{array}{l}\text { Sin infestación } \\
\text { visible }\end{array}$ \\
& $\begin{array}{l}\text { Menos de la mitad de } \\
\text { las ramas infestadas } \\
\text { por muérdago }\end{array}$ & $\begin{array}{l}1 \text { o } 2 \text { matas de } \\
\text { muérdago }\end{array}$ \\
& $\begin{array}{l}\text { Más de la mitad de } \\
\text { las ramas infestadas } \\
\text { por muérdago }\end{array}$ & $\begin{array}{l}\text { Más de 2 matas de } \\
\text { muérdago }\end{array}$ \\
\hline
\end{tabular}

TABLA 2. Clasificación del valor total de infestación por muérdago Cladocolea loniceroides en árboles y arbustos, dentro del parque Rodolfo Landeros Gallegos, Aguascalientes, México.

\begin{tabular}{cc}
\hline Valor total & Grado de infestación \\
\hline 0 & Nula \\
$1-2$ & Ligera \\
$3-4$ & Moderada \\
$5-6$ & Severa \\
\hline
\end{tabular}


Las aves registradas fueron identificadas a nivel de especie, por medio de guías de campo (Alderfer, 2006; Del Olmo y Roldán, 2013; Peterson y Chalif, 2008; Van-Perlo, 2006) y una guía digital (Merlin Bird ID, 2018). En su determinación se siguieron los nombres científicos aceptados por la American Ornithological Society [AOS] (2018). Se consideraron como potencialmente dispersoras a aquellas aves que fueron observadas alimentándose de los frutos de Cladocolea loniceroides.

\section{ResultadOS}

En el PRLG, Cladocolea loniceroides parasita a 16 especies (10 arbóreas y 6 arbustivas), pertenecientes a nueve familias (Tabla 3), 10 de las cuales son ornamentales introducidas como el rosa laurel (Nerium oleander L.) (Fig. 3A) y la granada (Punica granatum L.) (Fig. 3B) y seis son nativas, como el huizache (Acacia schaffneri (S. Watson) F.J. Herm) (Fig. 3C) y el gigante (Nicotiana glauca Graham) (Fig. 3D).

De los 4154 individuos observados, se contaron 2343 afectados, que equivalen a una incidencia de infestación total de poco más de $56 \%$. Entre las especies más afectadas destacan el álamo dólar (Populus alba L.) y el álamo común (P. canadensis Moench.), ambos con una incidencia de infestación de poco más de $80 \%$, seguidos por el sauce llorón (Salix babylonica L.) con $76.47 \%$ y el fresno (Fraxinus ubdei (Wenz.) Lingelsh.) con 59.14\% (Fig. 4).

El valor de infestación promedio fue de 1.6 y el mayor valor lo presentó Populus canadensis (2.42), seguido por Salix babylonica (2.29), Populus alba (2.1) y Fraxinus ubdei (1.79). Populus canadensis fue también la especie con el mayor valor de incidencia severa (30.91), seguida por Fraxinus ubdei y Salix babylonica (23.34 y 23.53, respectivamente). En total, existe una densidad de 33.47 árboles infestados por hectárea, la mayoría de los cuales corresponde a F. uhdei, con 23.86 (Tabla 3).

Se identificaron 18 especies de angiospermas libres de infestación por C. loniceroides (Tabla 4). Durante los conteos de árboles y arbustos, se corroboró que los individuos de estos taxones no estuvieran afectados por matas de muérdago.
Las aves que fueron observadas alimentándose de los frutos de $C$. loniceroides corresponden a siete especies pertenecientes a cinco familias (Tabla 5). Las más comunes son el zanate mayor (Quiscalus mexicanus), el pinzón mexicano (Haemorhous mexicanus), el tirano chibiú (Tyrannus vociferans), la paloma alas blancas (Zenaida asiatica) y el papamoscas cardenalito (Pyrocephalus rubinus) (Figs. 5A-D y 6). En las excretas de este último se encontraron semillas de C. loniceroides principalmente de enero a abril.

\section{DISCUSIÓN}

Aunque $C$. loniceroides es una planta nativa y endémica de México (Villaseñor, 2016), es probable que su presencia en la ciudad de Aguascalientes, México, se deba a una introducción causada por el hombre al plantar árboles infestados (Sandoval-Ortega y Siqueiros-Delgado, 2019), ya que ha sido localizada únicamente en un área verde urbana dentro de la ciudad y no en áreas forestales con vegetación nativa. Un caso similar ocurre en Ciudad de México, donde esta especie es también considerada introducida (Calderón de Rzedowski, 2001) por medio de árboles ornamentales infestados que fueron llevados de un vivero de Cuernavaca, Morelos (Cházaro Basáñez, Oliva Rivera, Ramón Farías y Vázquez García, 2005).

Estudios previos sugieren que la infestación por muérdago es mayor en áreas verdes urbanas que en bosques naturales (Cid-Villamil, 2006; Díaz-Limón, Cano-Santana y Queijeiro-Bolaños, 2016; Maruyama, Mendes-Rodiguez, Alves-Silva y Cunha, 2012). Esto puede deberse a que en las ciudades los suelos compactos y carentes de nutrientes, la contaminación atmosférica y los daños asociados al vandalismo, son factores que disminuyen la vitalidad del arbolado y lo hacen vulnerable al ataque de plagas y enfermedades, como la infestación por muérdago (CidVillamil y Bye, 1998; Maruyama et al., 2012; ZaragozaHernández et al., 2015). Es posible que, en comparación con la vegetación árida natural distribuida en la meseta central del estado de Aguascalientes (Siqueiros-Delgado et al., 2017), en donde se sitúa la ciudad del mismo nombre, estos factores sumados al riego continuo generen un ambiente más adecuado para el desarrollo de C. loniceroides causando que este muérdago, al menos por ahora, se encuentre restringido al PRLG. 
TABLA 3. Grado de infestación por Cladocolea loniceroides en especies hospederas presentes en el parque Rodolfo Landeros Gallegos, Aguascalientes, México.

\begin{tabular}{|c|c|c|c|c|c|c|c|c|c|c|c|c|c|c|}
\hline \multirow{2}{*}{ Especie } & \multirow{2}{*}{ Hábito } & \multirow{2}{*}{$\begin{array}{c}\text { Individuos } \\
\text { totales }\end{array}$} & \multirow{2}{*}{$\begin{array}{l}\text { Individuos } \\
\text { infestados }\end{array}$} & \multicolumn{7}{|c|}{ Valor de infestación } & \multirow{2}{*}{$\begin{array}{l}\text { Valor de } \\
\text { infestación } \\
\text { promedio }\end{array}$} & \multicolumn{2}{|c|}{ Incidencia } & \multirow{2}{*}{ Densidad } \\
\hline & & & & 0 & 1 & 2 & 3 & 4 & 5 & 6 & & ligera & Severa & \\
\hline
\end{tabular}

\section{Apocynaceae}

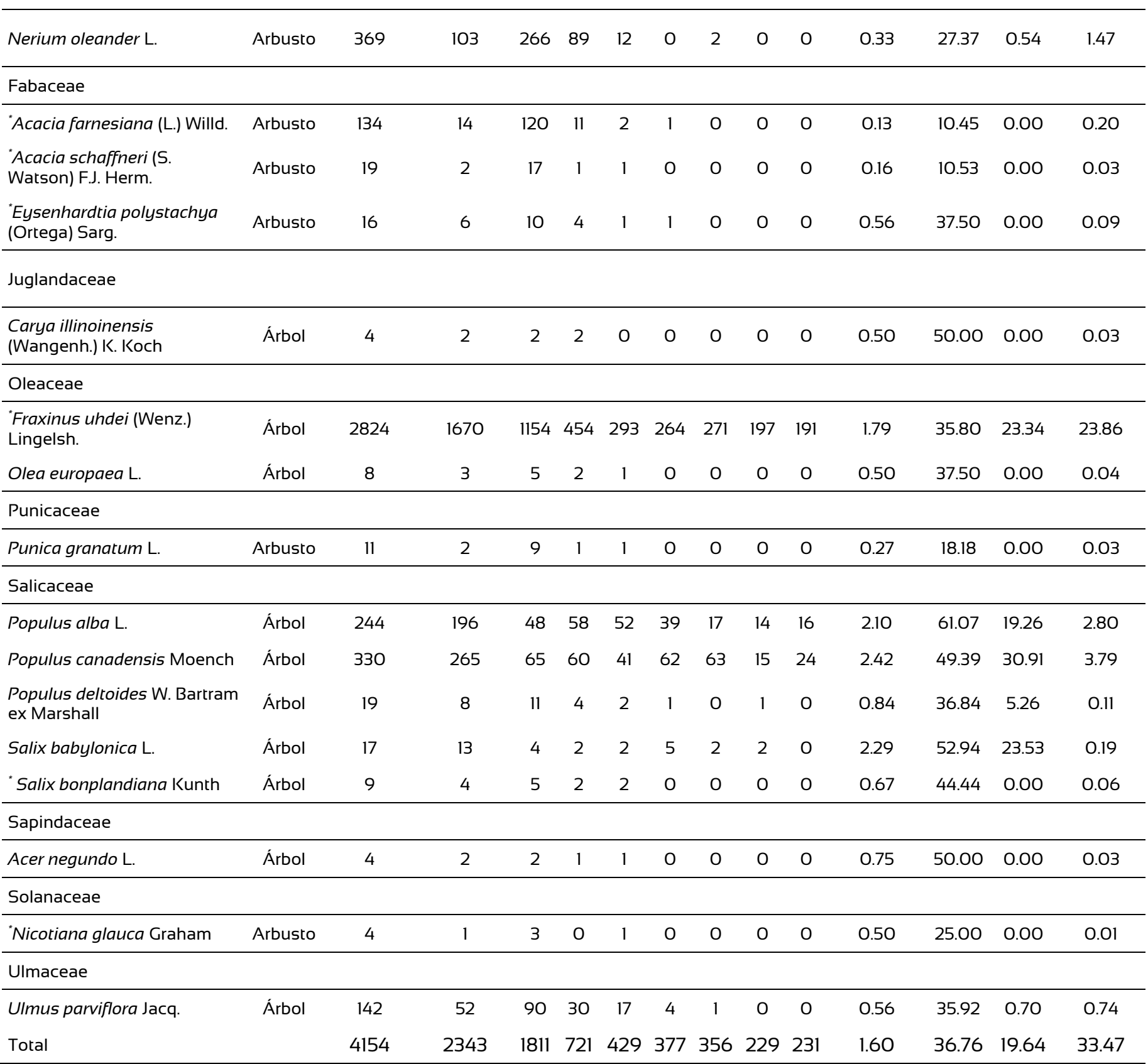

$*$ Nativo. 


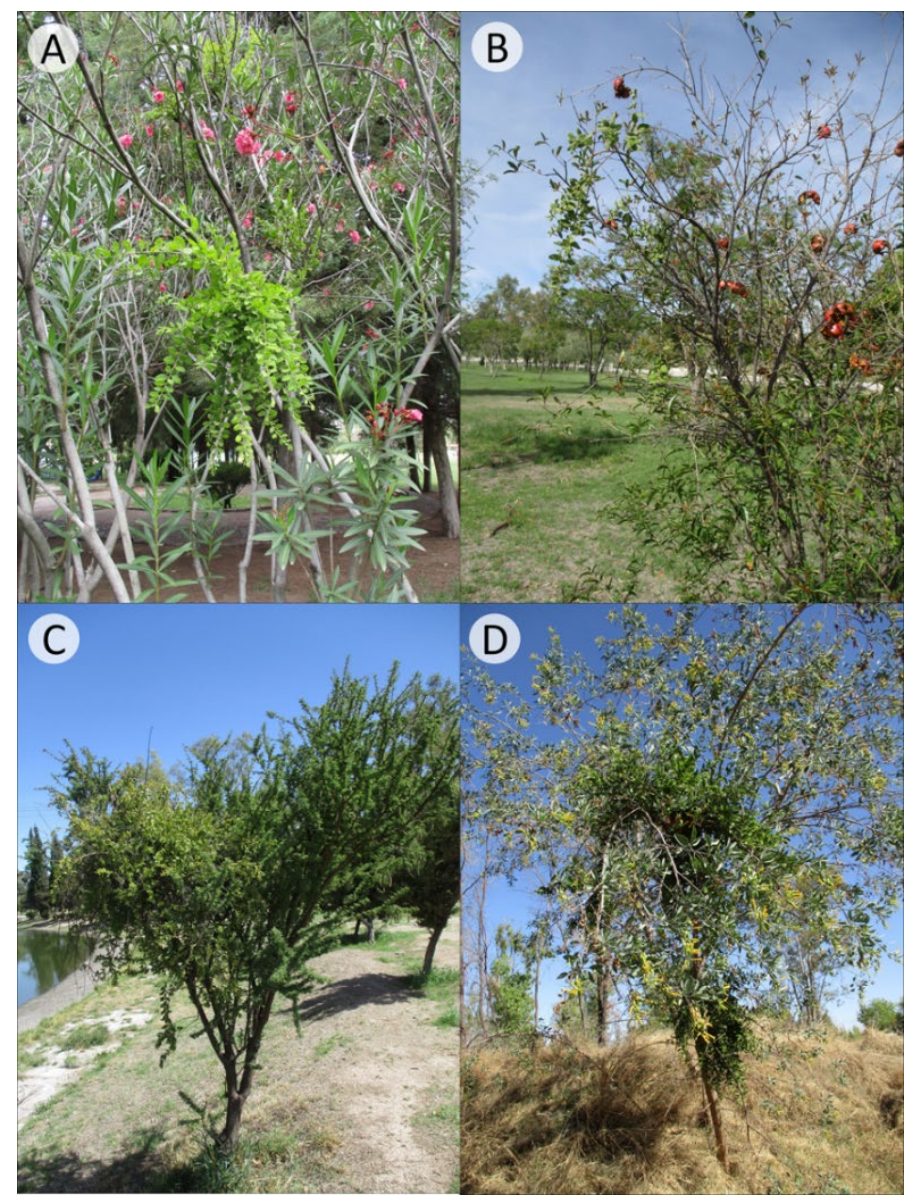

FIgURA 3. Algunas especies afectadas por Cladocolea loniceroides, presentes en el parque Rodolfo Landeros Gallegos, Aguascalientes, México. A: Rosa laurel (Nerium oleander). B: Granada (Punica granatum). C: Huizache (Acacia schaffnern). D: Gigante (Nicotiana glauca).

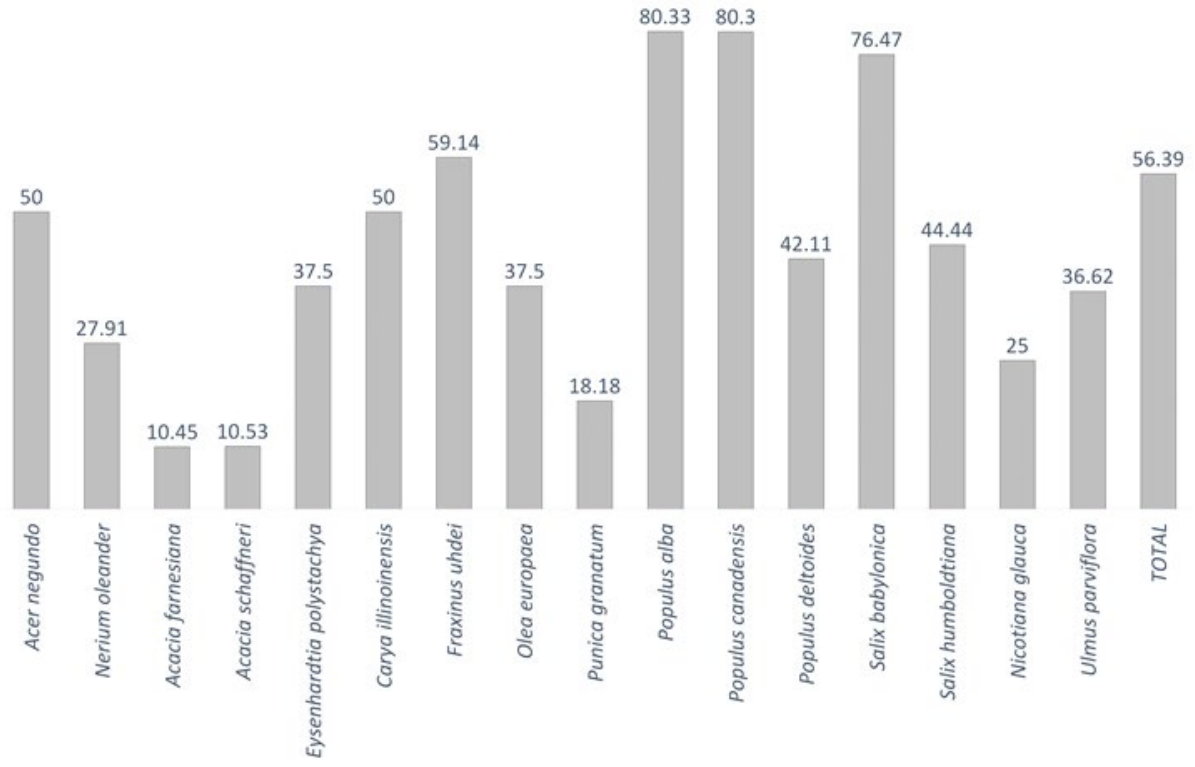

FIGURA 4. Incidencia de infestación por el muérdago Cladocolea loniceroides en especies arbustivas y arbóreas presentes en el parque Rodolfo Landeros Gallegos, Aguascalientes, México. 
TABLA 4. Especies de angiospermas arbustivas o arbóreas presentes en el parque Rodolfo Landeros Gallegos libres de infestación por el muérdago Cladocolea loniceroides.

\begin{tabular}{lllc}
\hline \multicolumn{1}{c}{ Familia } & Especie & Nombre común & Hábito \\
\hline Altingiaceae & Liquidambar styraciflua L. & Liquidámbar & Árbol \\
& Schinus molle L. & Pirul & Árbol \\
Anacardiaceae & Schinus terebinthifolias Raddi & Pirul brasileño & Árbol/arbusto \\
Bignoniaceae & Jacaranda mimosifolia D. Don & Jacaranda & Árbol \\
Casuarinaceae & Casuarina equisetifolia L. & Casuarina & Árbol \\
Fabaceae & Prosopis laevigata (Humb. \& Bonpl. ex Willd.) M.C. Johnst. & Mezquite & Árbol/arbusto \\
& Erythrina americana Mill. & Colorín & Árbol \\
Fagaceae & Quercus resinosa Liebm. & Encino blanco & Árbol \\
& Quercus rugosa Née & Encino & Árbol \\
Meliaceae & Melia azedarach L. & quiebrahacha & Paraíso \\
Moraceae & Ficus retusa L. & Laurel de la india & Árbol \\
& Eucalyptus camaldulensis Dehnh. & Eucalipto & Árbol \\
Myrtaceae & Eucalyptus cinerea F.Muell. ex Benth. & Eucalipto dólar & Árbol \\
& Psidium guajava L. & Guayabo & Árbol/arbusto \\
Oleaceae & Ligustrum lucidum W.T. Aiton & Trueno & Árbol \\
Proteaceae & Grevillea robusta A. Cunn. ex R. Br. & Grevilea & Árbol \\
Rosaceae & Eriobotrya japonica (Thunb.) Lindl. & Níspero & Árbol \\
Tamaricaceae & Tamarix gallica L. & Tamarisco & Árbol \\
\hline
\end{tabular}

TABLA 5. Aves dispersoras de las semillas del muérdago Cladocolea loniceroides dentro del parque Rodolfo Landeros Gallegos, Aguascalientes, México.

\begin{tabular}{cccc}
\hline Familia & Especie & Nombre común & Estacionalidad' $^{\prime}$ \\
\hline Columbidae & Zenaida asiatica & Paloma alas blancas & $\mathrm{R}$ \\
Fringillidae & Haemorhous mexicanus & Pinzón mexicano & $\mathrm{R}$ \\
Icteridae & Quiscalus mexicanus & Zanate mayor & $\mathrm{R}$ \\
Parulidae & Setophaga coronata & Chipe rabadilla amarilla & $\mathrm{R}$ \\
Tyranidae & Pitangus sulphuratus & Luis bienteveo & $\mathrm{R}$ \\
& Pyrocephalus rubinus & Papamoscas cardenalito & $\mathrm{R}$ \\
\hline
\end{tabular}




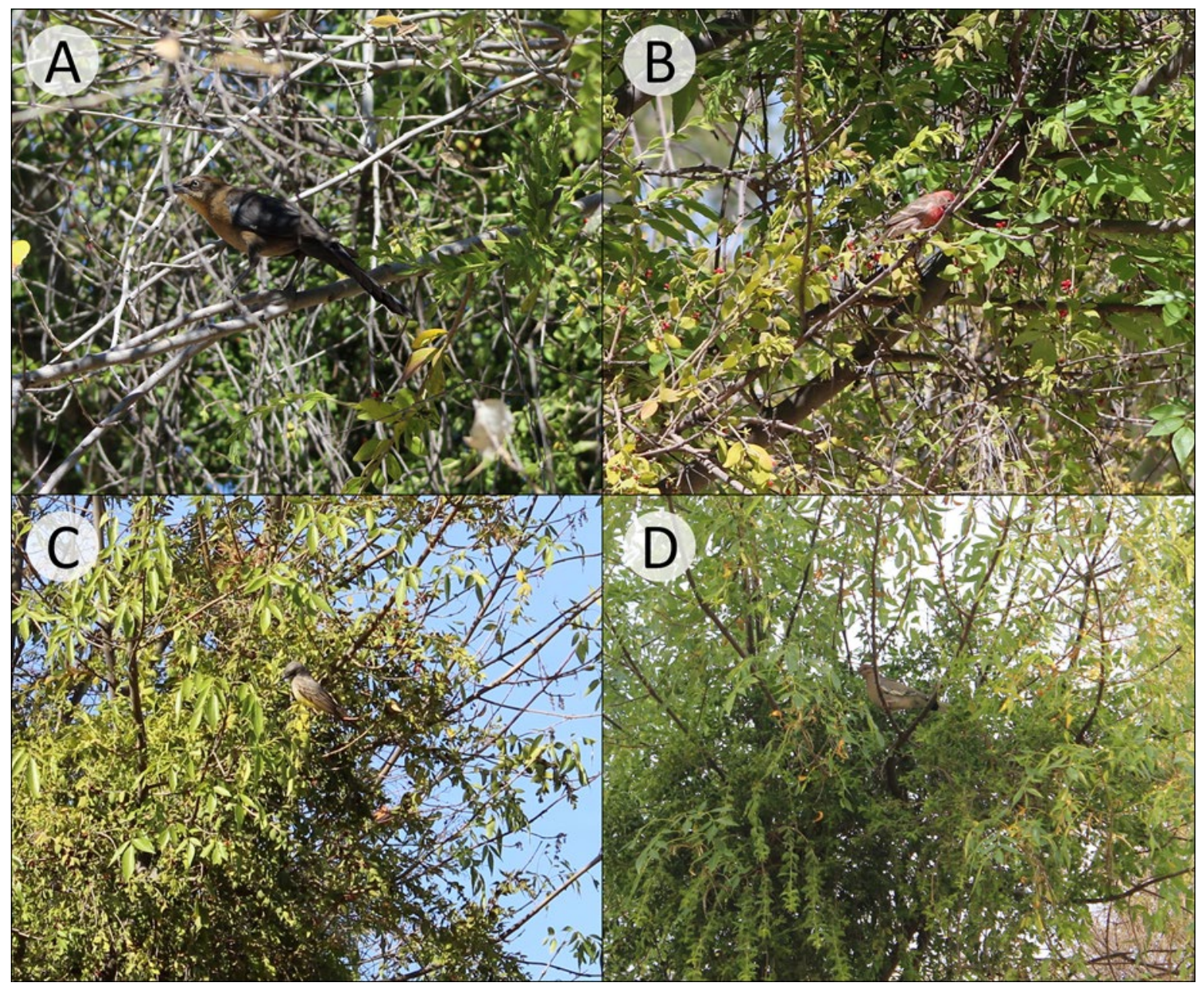

Figura 5. Aves observadas alimentándose de frutos de Cladocolea loniceroides en el parque Rodolfo Landeros Gallegos, Aguascalientes, México. A: Zanate mayor (Quiscalus mexicanus). B: Pinzón mexicano (Haemorbous mexicanus). C: Tirano chibiú (Tyrannus vociferans). D: Paloma de alas blancas (Zenaida asiatica).
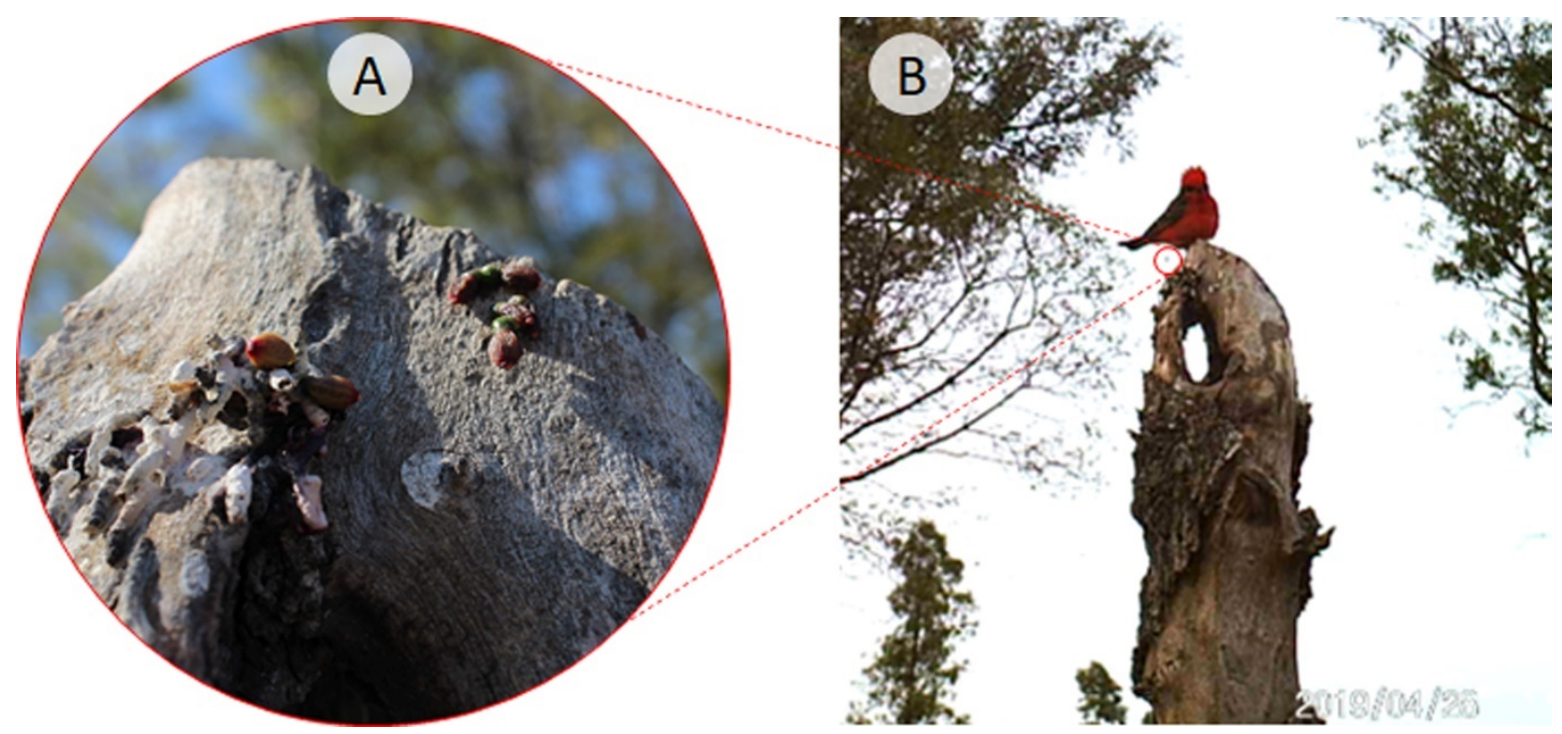

Figura 6. A. Excretas de papamoscas cardenalito (Pyrocephalus rubinus) con semillas de Cladocolea loniceroides. B. Papamoscas cardenalito (Pyrocephalus rubinus), en el parque Rodolfo Landeros Gallegos, Aguascalientes, México. 
Para estimar la severidad de la infestación por C. loniceroides, algunos autores (Alvarado-Rosales \& Saavedra-Romero, 2005; Arriola-Padilla et al., 2013) han empleado el sistema de calificación de cinco niveles utilizado por Pérez-Laorga, Alguacil y Montero (2001) en Viscum album L. y adaptada para C. loniceroides (Alvarado-Rosales et al., 2003). No obstante, este sistema presenta ciertas desventajas, aún con las modificaciones hechas por Alvarado-Rosales et al. (2003), para evaluar la infestación por C. loniceroides. Lo anterior, debido a que fue diseñado para especies de pinos (Pinus spp.), los cuales son plantas siempre verdes, cuyas hojas pueden persistir más de dos años (Earle, 2020). Por esto, dos de los cinco niveles consideran la dominancia del follaje del hospedero respecto al follaje de las matas de muérdago que lo parasitan, lo que resulta difícil de determinar en especies caducifolias; además, el nivel cinco corresponde a árboles muertos, los cuales comúnmente carecen de estructuras para poder ser identificados.

Por las razones anteriores se optó por utilizar el sistema de seis niveles propuesto por Hawksworth (1977) con algunas modificaciones. A diferencia del sistema de Perez-Laorga et al. (2001), este toma en cuenta el número de ramas infestadas en lugar de la abundancia del follaje del hospedero, por lo que es más adecuado para especies caducifolias, además no considera a los individuos muertos. Sin embargo, el sistema de Hawksworth (1977) no se recomienda para árboles con menos de $3 \mathrm{~m}$ de altura, por lo que lo que fue necesario hacer modificaciones, que se basaron en las sugerencias que el mismo autor menciona al describir las limitaciones de su sistema. Se toma en cuenta para arbustos y árboles menores de $3 \mathrm{~m}$ el número de matas de muérdago en lugar de la proporción de ramas afectadas, la cual sí se considera en el caso de árboles mayores a $3 \mathrm{~m}$ y de ramificación densa.

Aunque el sistema de Hawksworth (1977) está diseñado para evaluar la infestación por muérdagos enanos (Arceuthobium), ya ha sido modificado para ser aplicado en especies arbóreas afectadas por los géneros Struthanthus, Psittacanthus y Phoradendron (Bello, 1984; Howell y Mathiasen, 2004), mostrando que, con base en la proporción de ramas afectadas se puede valorar la infestación por muérdagos, tanto enanos como verdaderos.

La presencia de $C$. loniceroides representa una amenaza tanto para la vegetación nativa como para especies ornamentales y cultivos dentro del estado, pues se detectó que afecta especies de importancia agrícola como la granada (Punica granatum L.), el nogal (Carya illinoinensis (Wangenh.) K. Koch) y el olivo (Olea europaea L.). Además, se ha reportado que parasita árboles de aguacate (Persea americana Mill.), durazno (Prunus persica (L.) Batsch), cítricos (Citrus spp.) y capulín (Prunus serotina Ehrh.) (Vazquez-Collazo et al., 2006).

Las especies más afectadas por C. loniceroides dentro del área de estudio son el álamo común (Populus canadensis), el álamo dólar (P. alba) y el fresno (Fraxinus ubder), que se utilizan como plantas ornamentales en áreas verdes de la ciudad de Aguascalientes (De la Cerda-Lemus y GonzálezAdame, 2009; Semadesu, 2013). El fresno (F. ubdeı) se reporta como hospedero de esta misma especie de muérdago en otros sitios del país (Arriola-Padilla et al., 2013; Díaz-Limón et al., 2016; Vazquez-Collazo et al., 2006) y junto con el varaduz (Eysenhardtia polystachya (Ortega) Sarg.), especie nativa del estado que también presenta infestación en el PRLG, es una de las más afectadas por C. loniceroides en otras áreas verdes urbanas (Díaz-Limón et al., 2016).

El fresno (F. ubdeə), también se encuentra de manera silvestre en el estado, como un elemento de bosque de galería junto con Salix bonplandiana Kunth (SiqueirosDelgado, Rodríguez-Avalos, Martínez-Ramírez, SierraMuñoz y García-Regalado, 2017), otra especie nativa afectada por C. loniceroides, tanto en el área de estudio como en otros sitios de México (Alvarado Rosales y Saavedra Romero, 2005; Arriola Padilla et al., 2013). El bosque de galería es uno de los tipos de vegetación menos extensos en Aguascalientes, pues ocupa $0.35 \%$ del estado y se encuentra en una situación relictual, con problemas de supervivencia (Siqueiros-Delgado, Rodríguez-Avalos, Martínez-Ramírez y Sierra-Muñoz, 2016; Siqueiros-Delgado et al., 2017), por lo que es el tipo de vegetación que podría verse más afectado por C. loniceroides, si la distribución de esta planta hemiparásita se extiende más allá del PRLG. 
Entre las especies no afectadas presentes en el PRLG se encuentran el colorín (Erythrina americana Mill.), el laurel de la india (Ficus retusa L.) y el liquidámbar (Liquidambar styraciflua L.), que ya se han señalado como libres de infestación por muérdago en otros estudios realizados en el país (De la Paz Pérez-Olvera et al., 2006). Sucede lo contrario con la rosa laurel (Nerium oleander L.), que se ha registrado como libre de infestación por muérdago en otros estudios (De la Paz Pérez-Olvera et al., 2006), pero dentro del PRLG se observaron varios individuos con matas de $C$. loniceroides bien desarrolladas.

De igual manera, algunos autores (Arriola-Padilla et al., 2013; Calderón de Rzedowski, 2001; Kuijt, 1975) han identificado al trueno (Ligustrum lucidum W.T. Aiton) como hospedero de C. loniceroides. No obstante, en el área de estudio, en donde existen áreas forestadas únicamente con individuos de esta especie, no se detectaron árboles afectados por muérdago. Lo mismo ocurre para Quercus rugosa Née, que es mencionada como uno de los hospederos con mayor frecuencia e intensidad de infestación en otra área verde urbana en el país (Díaz-Limón et al., 2016), pero en el PRLG, al igual que las demás especies de este género como Q. resinosa Liebm., está libre de infestación.

Las aves son los principales dispersores de semillas de muérdagos verdaderos (Mathiasen et al., 2008; Watson, 2001), dicha dispersión ocurre por medio de tres mecanismos: 1) la defecación, 2) la regurgitación y 3) el abandono de las semillas pegajosas sobre las ramas (Stevens, 2001), por lo que puede considerarse a toda ave que se alimenta de frutos de muérdago como potencial dispersor de sus semillas.

Un estudio de aves dispersoras realizado para otra especie de la familia Loranthaceae (López-de Buen y Ornelas, 2001) sugiere que el muérdago utiliza una estrategia de alta inversión en nutrimentos para atraer a dispersores durante el tiempo de escasez de frutos. Este podría ser también el caso de $C$. loniceroides, ya que las especies que se alimentan de sus frutos corresponden en su mayoría a especies generalistas, que utilizan esta fuente de alimento durante el invierno, cuando hay poca disponibilidad de otros frutos, semillas e insectos. Además, las aves generalistas poseen otras fuentes de alimento, por lo que pueden visitar áreas libres de infestación, contribuyendo potencialmente al incremento de la dispersión del muérdago (Rawsthorne, Watson y Roshier, 2011).

Entre las especies observadas alimentándose de frutos de C. loniceroides en el PRLG, se encuentra la paloma alas blancas (Zenaida asiatica) y el zanate mayor (Quiscalus mexicanus), ambas especies comunes en zonas urbanas y agrícolas (Del Olmo y Roldán, 2013; Kaufman, 1996). El zanate mayor ha sido considerado como una de las principales aves que dispersan muérdago en la ciudad de México (Comisión Nacional Forestal [Conafor], 2010).

Otras especies que han sido identificadas anteriormente como dispersoras de muérdago son el tirano chibiú (Tyrannus vociferans), para Psittacanthus auriculatus (Oliv.) Eichler y P. calyculatus (DC.) G. Don (Díaz-Infante, Lara, Arizmendi, Eguiarte y Ornelas, 2016) y el luis bienteveo (Pitangus sulphuratus), en el caso de Phoradendron affine (Pohl ex DC.) Engl. \& K. Krause (Maruyama et al., 2012).

Las aves, como el resto de los animales, no pueden sintetizar pigmentos carotenoides y deben obtenerlos de sus alimentos (Hill, 1992; Salazar-González, 2000) acumulándolos en el cuerpo y en el plumaje (SalazarGonzález, 2000). Una de las especies para la que se han estudiado las bases bioquímicas para la coloración, es el pinzón mexicano (Haemorbous mexicanus) (Hill, 1992), cuyo plumaje con coloración rojiza contiene carotenoides como $\beta$-caroteno, isocriptoxantina y equinenona (Brush y Power, 1976). Los carotenoides son los responsables de muchos casos de la coloración roja, naranja y amarilla en frutos (Namitha y Negi, 2010). Por esto, es posible que los frutos rojos de C. loniceroides sean una fuente de nutrientes y pigmentos para los pinzones mexicanos que se alimentan de ellos en el PRLG.

Por otro lado, el papamoscas cardenalito (Pyrocephalus rubinus) es un ave de la que se sabe poco acerca de su dieta (Ellison, Wolf y Jones, 2009; Kaufman, 1996). Algunos autores mencionan que al parecer consiste solo de artrópodos (Billerman, Keeney, Rodewald y Schulenberg, 2020; Ellison et al., 2009; Kaufman, 1996; MacGregor-Fors, 
2010), mientras que otros señalan que también pueden consumir pequeños frutos (Maruri-Aguilar, García-Valdés y Pineda-López, 2013). En el presente estudio se corrobora que Pyrocephalus rubinus incluye dentro de su dieta frutos de C. Loniceroides, y dentro del PRLG es común encontrar entre sus excretas semillas de este muérdago, principalmente durante el invierno y principios de primavera. Es posible que al igual que el pinzón mexicano, el papamoscas cardenalito complemente su alimentación con los frutos de C. loniceroides para obtener los carotenoides necesarios para el característico color rojo en su plumaje.

Por último, el chipe rabadilla amarilla (Setophaga coronata) es una especie que anida en Estados Unidos y Canadá y en invierno migra a territorio mexicano; en el verano se alimenta principalmente de insectos, pero durante la migración y el invierno consume una amplia variedad de frutos (Billerman et al., 2020). Existen reportes de que las aves migratorias que se alimentan de los frutos de muérdago pueden transportar sus semillas a grandes distancias (Barlow y Schodde, 1993; Cházaro-Basáñez y Oliva-Rivera, 1991). Debido a lo anterior, cabe la posibilidad de que el chipe rabadilla amarilla pueda transportar semillas en su tracto digestivo durante su camino de regreso a sus sitios de anidación, contribuyendo a la expansión de la distribución de $C$. loniceroides hacia el norte del país.

\section{CONCLUSIONES}

Debido a que más de la mitad de los individuos de las especies hospederas de $C$. loniceroides en el PRLG muestran infestación por este muérdago, se considera como grave el estado fitosanitario de la zona de estudio. En este caso en particular es recomendable no plantar más álamo dólar (Populus alba), álamo común (P. canadensis), sauce llorón (Salix babylonica) y fresno (Fraxinus ubder), ya que son las especies más susceptibles de ser infestadas por $C$. loniceroides. Se recomienda reemplazar los árboles muertos o con infestación severa, por especies nativas como el mezquite (Prosopis laevigata), el encino blanco (Quercus resinosa) y el quiebrahacha (Q. rugosa) que han mostrado no ser afectadas por C. loniceroides dentro del área de estudio o con gimnospermas como el pino piñonero (Pinus cembroides).

Pese a ser una especie dañina para el arbolado dentro del área de estudio, C. loniceroides representa también un recurso importante para la avifauna local, ya que es una fuente de alimento disponible durante el invierno, cuando otros recursos escasean. Por lo anterior, deben incluirse plantas que sustituyan la fuente de alimento que $C$. loniceroides representa para las aves del PRLG. Es el caso de Pithecellobium dulce (Roxb.) Benth. o Forestiera phillyreoides (Benth.) Torr., que son especies nativas y, al menos por ahora, no han sido reportadas como hospederas de $C$. loniceroides.

El reemplazo paulatino de especies susceptibles a la infestación por muérdago, aunado a la poda sanitaria, disminuirán la presencia de $C$. Loniceoides en esta área verde urbana hasta erradicarla, ya que actualmente representa una amenaza para otras zonas con vegetación dentro de la ciudad de Aguascalientes. También es recomendable que los árboles que se pretendan introducir al parque y que sean procedentes de vivero, sean inspeccionados en busca de cualquier señal de infestación por C. loniceroides u otra especie de muérdago antes de ser plantado, para evitar una nueva introducción.

Es necesario realizar un estudio más detallado sobre las aves que consumen el fruto de $C$. loniceroides, para determinar si las semillas excretadas o regurgitadas son viables y comprobar así que estas especies de aves son dispersoras de dicho muérdago verdadero.

\section{RefERENCIAS}

Alderfer, J. (2006). Field Guide to the Bird of North America (5a ed.). Estados Unidos: National Geographic Society.

Alvarado-Rosales, R. D., Equihua-Martínez, A., Estrada-Venegas, E., Franco-Maldonado, V., González-Monzón, A. U., \& SaavedraRomero, L. de L. (2003). The Cladocolea genus in the Mexican City Basin and its management. 1st Joint Meeting of the 12th National Symposium on Forest Parasites and the 54th Western Forest Insect Work Conference (WFIWC), 10. México.

Alvarado-Rosales, D., \& Saavedra-Romero, L. de L. (2005). El Género Cladocolea (Loranthaceae) en México: Muérdago Verdadero o 
Injerto. Revista Chapingo Serie Ciencias Forestales y Del Ambiente, 11(1), 5-9.

American Ornithological Society [AOS]. (2018). Checklist of North and Middle American Birds. Recuperado de http://checklist.americanornithology.org/

Arriola-Padilla, V. J., Velasco-Bautista, E., Hernández-Tejeda, T., González-Hernández, A., \& Romero-Sánchez, M. E. (2013). Los muérdagos verdaderos del arbolado de la ciudad de México. Revista Mexicana de Ciencias Forestales, 4(19), 34-45.

Aukema, J. E., \& Martinez del Rio, C. (2002). Where does a fruit-eating bird deposit mistletoe seeds? Seed deposition patterns and an experiment. Ecology, 83(12), 3489-3496. doi: 10.1890/00129658(2002)083[3489:WDAFEB]2.0.CO;2

Barlow, B. A., \& Schodde, R. (1993). Bird dispersal of loranthaceous mistletoes to remote pacific islands: symbiosis in default. Beaufortia, 43(8), 124-124.

Bello González, M. A. 1984. Estudio de muérdagos (Loranthaceae) en la región Tarasca, Michoacán. Boletín 102. D.F., México: INIFAP. En la publicación original el apellido es Bello González ¿`se respeta aquí? si es así, habría que cambiar en el texto donde aparece el autor. No me fue posible poner esta nota como comentario.

Billerman, S. M., Keeney, B. K., Rodewald P. G., Schulenberg, T. S. (2020). Birds of the World. Cornell Laboratory of Ornithology. Ithaca, Nueva York, Estados Unidos. Recuperado de https://birdsoftheworld.org

Brush, A. H., \& Power, D. M. (1976). House Finch pigmentation: Carotenoid metabolism and the effect of diet. The Auk, 93, 725739 .

Calderón de Rzedowski, G. (2001). Loranthaceae. En G. Calderón \& J. Rzedowski (Eds.), Flora del Valle de México (pp. 106) (2 $2^{\mathrm{a}}$ ed.). Xalapa, México: Instituto de Ecología, A.C. y CONABIO.

Cházaro Basáñez, M. J., \& Oliva Rivera, H. (1991). Dendrophthora costaricensis Urban (Loranthaceae), un nuevo registro para la Flora de México. Acta Botanica Mexicana, 13, 31-39. doi: 10.21829/abm13.1991.606

Cházaro Basáñez, M. J., Oliva Rivera, H., Ramón Farías, F., \& Vázquez García, J. A. (2005). Cladocolea oligantha (Loranthaceae) un nuevo registro para Veracruz, México, y datos generales sobre este taxón. Polibotánica, 20, 1-15.

Cid-Villamil, R. M. (2006). Biologia del desarrollo de Cladocolea loniceroides (Van Tieghem) Kuijt (Loranthaceae). Tesis de Doctorado, Universidad Nacional Autónoma de México, D.F., México.
Cid-Villamil, R. M., \& Bye, R. A. (1998). Site conditions of an urban wooded area of Mexico City that hosts Cladocolea loniceroides Van Tieghem (Loranthaceae). Selbyana, 19(2), A 272.

Comisión Nacional Forestal [Conafor]. (2010). El muérdago en el arbolado urbano. Recuperado de http://www.conafor.gob.mx:8080/ documentos/docs/18/19027_Muérdago en arbolado urbano.pdf

De la Cerda-Lemus, M. (1989). Encinos de Aguascalientes. Aguascalientes, México: Universidad Autónoma de Aguascalientes.

De la Cerda-Lemus, M. (2000). Álamos y sauces del estado de Aguascalientes. Investigación y Ciencia, 23, 17-24.

De la Cerda-Lemus, M., \& González-Adame, G. (2009). Plantas ornamentales de la ciudad de Aguascalientes. Aguascalientes, México: Universidad Autónoma de Aguascalientes.

De la Paz Pérez-Olvera, C., Ceja-Romero, J., \& Vela-Ramírez, G. (2006). Árboles y muérdagos: una relación que mata. Contactos, 59, 28-34.

Del Olmo, L. G., \& Roldán, E. (2013). Aves Comunes de la Ciudad de México. México: Bruja de Monte, CONABIO.

Díaz-Infante, S., Lara, C., Arizmendi, M. del C., Eguiarte, L. E., \& Ornelas, J. F. (2016). Reproductive ecology and isolation of Psittacanthus calyculatus and P. auriculatus mistletoes (Loranthaceae). PeerJ, 4(e2491), 1-29. doi: 10.7717/peerj.2491

Díaz-Limón, M. P., Cano-Santana, Z., \& Queijeiro-Bolaños, M. E. (2016). Mistletoe infection in an urban forest in Mexico City. Urban Forestry \& Urban Greening, 17, 126-134. doi: 10.1016/j.ufug.2016.04.004

Earle, C. J. 2020. The Gymnosperm Database. Recuperado de https://www.conifers.org/index.php

Ellison, K., Wolf, B. O., \& Jones, S. L. (2009). Vermilion Flycatcher (Pyrocephalus rubinus), version 2.0. En Poole, A. F. (Ed.), The Birds of North America. Ithaca, Estados Unidos: Cornell Lab of Ornithology. doi: 10.2173/bna.484

Engelmann, G. (1986). Instructions for the collection and preservation of botanical specimens. Annals of the Missouri Botanical Garden, 73, 504-507.

Geils, B. W., Cibrian-Tovar, J., \& Moody, B. (2002). Mistletoes of North American Conifers. Estados Unidos: USDA Forest Service.

Hawksworth, F. G. (1977). The 6-Class Dwarf Mistletoe Rating System. Estados Unidos: USDA Forest Service.

Hill, G. (1992). Proximate basis of variation in carotenoid pigmentation in male hause finches. The Auk, 109(1), 1-12.

Howell, B. E., \& Mathiasen, R. L. (2004). Growth impacts of Psittacanthus angustifolius Kuijt on Pinus oocarpa Schiede in Honduras. Forest Ecology and Management, 198, 75-88. doi:10.1016/j.foreco.2004.03.047 
Judd, W. S., Campell, C. S., Kellogg, E. A., Stevens, P. F., \& Donoghue, M. J. (2016). Plant Systematics: A Phylogenetic Approach (4a ed.). Sunderland, Estados Unidos: Sinauer Associates.

Kaufman, K. (1996). Lives of north american birds. Estados Unidos: Houghton Mifflin Harcourt.

Kuijt, J. (1975). The Genus Cladocolea (Loranthaceae). Journal of the Arnold Arboretum, 56(3), 265-335.

Kuijt, J. (2011). Pulling the skeleton out of the closet: resurrection of Phthirusa sensu Martius and consequent revival of Passovia (Loranthaceae). Plant Diversity and Evolution, 129(2), 159-211. doi: 10.1127/1869-6155/2011/0129-0049

López-de Buen, L., \& Ornelas, J. F. (2001). Seed Dispersal of the Mistletoe Psittacanthus schiedeanus by Birds in Central Veracruz, Mexico. Biotropica, 33(3), 487-494.

MacGregor-Fors, I. (2010). Guía de aves del bosque Los Colomos. México: Conabio.

Maruri-Aguilar, B., García-Valdés, A. I., \& Pineda-López, R. (2013). Las aves del jardín botánico regional de cadereyta: Una presencia interpretada. México: Consejo de Ciencia y Tecnología del Estado de Querétaro, Jardín Botánico Regional de Cadereyta.

Maruyama, P. K., Mendes-Rodrigues, C., Alves-Silva, E., \& Cunha, A. F. (2012). Parasites in the neighbourhood: Interactions of the mistletoe Phoradendron affine (Viscaceae) with its dispersers and hosts in urban areas of Brazil. Flora - Morphology, Distribution, Functional Ecology of Plants, 207(10), 768-773. doi: 10.1016/j.flora.2012.08.004

Mathiasen, R. L., Nickrent, D. L., Shaw, D. C. \& Watson, D. M. (2008). Mistletoes: pathology, systematics, ecology and management. Plant Disease, 92, 988-1006. doi: 10.1094/PDIS-92-7-0988

Merlin Bird ID. (2018). Cornell Laboratory of Ornithology. Ithaca, Nueva York, Estados Unidos. Recuperado de https://merlin.allaboutbirds.org/

Namitha, K. K., \& Negi, P. S. (2010). Chemistry and Biotechnology of Carotenoids. Critical Reviews in Food Science and Nutrition, 50(8), 728760. doi: https://doi.org/10.1080/10408398.2010.499811

Pérez-Laorga, E., Alguacil, F., \& Montero, J. L. (2001). Distribución y características de la población del muérdago (Viscum album austriacum) en la comunidad valenciana. Valencia, España: Conselleria de Medio Ambiente.

Peterson, R. T., \& Chalif, E. L. (2008). Aves de México. México: Editorial Diana.

Rawsthorne, J., Watson, D. M., \& Roshier, D. A. (2011). Implications of movement patterns of a dietary generalist for mistletoe seed dispersal. Austral Ecology, 36, 650-655.
Salazar-González, M. (2000). Carotenoides: distribución en el mundo vegetal y animal. Contactos, 37, 60-68.

Sandoval-Ortega, M. H., \& Siqueiros-Delgado, M. E. (2019). Cladocolea loniceroides, un nuevo registro para la flora de Aguascalientes, México. Investigación y Ciencia de La Universidad Autónoma de Aguascalientes, 27(78), 51-54.

Secretaría del Medio Ambiente y Desarrollo Sustentable [Semadesu]. (2013). Manual de reforestación y manejo de áreas verdes urbanas del municipio de Aguascalientes. Aguascalientes, México: Semadesu.

Secretaría de Medio Ambiente y Recursos Naturales [Semarnat]. (2019). Informe de la Situación del Medio Ambiente en México, Edición 2018. Ciudad de México, México: Semarnat.

Siqueiros-Delgado, M. E. (1996). Leguminosas de Aguascalientes. Aguascalientes, México: Universidad Autónoma de Aguascalientes.

Siqueiros-Delgado, M. E., Rodríguez-Avalos, J. A., Martínez-Ramírez, J., \& Sierra-Muñoz, J. C. (2016). Situación actual de la vegetación del estado de Aguascalientes, México. Botanical Sciences, 94(3), 455470. doi: 10.17129/botsci.466

Siqueiros-Delgado, M. E., Rodríguez-Avalos, J. A., Martínez-Ramírez, J., Sierra-Muñoz, J. C., \& García-Regalado, G. (2017). Vegetación del Estado de Aguascalientes (1a ed.). Aguascalientes, México: Universidad Autónoma de Aguascalientes, CONABIO.

Start, T. (2011). Some Observations on an Urban Mistletoe Dendrophthoe pentandra (L.) Miq. (Loranthaceae) in Thailand. Natural History Bulletin of the Siam Society, 57, 81-86.

Stevens, P. C. (2001). Angiosperm Phylogeny Website. Recuperado de http://www.mobot.org/MOBOT/research/APweb/

Tercero-Bucardo, N., \& Rovere, A. E. (2010). Patrones de dispersión de semillas y colonización de Misodendrum punctulatum (Misodendraceae) en un matorral postfuego de Nothofagus antarctica (Nothofagaceae) del noroeste de la Patagonia. Revista Chilena de Historia Natural 83, 375-386. doi: 10.4067/S0716078X2010000300005

Van-Perlo, B. (2006). A field guide to the birds of Mexico and Central America. Londres, Inglaterra: Harper Collins Publisher.

Vazquez-Collazo, I., Villa-Rodríguez, A., \& Madrigal-Huendo, S. (2006). Los Muérdagos (Loranthaceae) en Michoacán. Michoacán, México: Sagarpa, Inifap.

Villaseñor, J. L. (2016). Checklist of the native vascular plants of Mexico. Revista Mexicana de Biodiversidad, 87, 559-902. doi: 10.1016/j.rmb.2016.06.017 
Watson, D. M. (2001). Mistletoe - A Keystone Resource in Forests and Woodlands Worldwide. Annual Review of Ecology and Systematics, 32(1), 219-249. doi: 10.1146/annurev.ecolsys.32.081501.114024

Zaragoza-Hernández, A. Y., Cetina-Alcalá, V. M., López-López, M. Á., Chacalo-Hilú, A., de la Isla-de Bauer, M. de L., Alvarado-Rosales, D., \& González-Rosas, H. (2015). Identificación de daños en el arbolado de tres parques del Distrito Federal. Revista Mexicana de Ciencias Forestales, 6(32), 63-82.

Manuscrito recibido el 5 de diciembre de 2019

Aceptado el 10 de junio de 2020

Publicado el 9 de julio de 2021
Este documento se debe citar como:

Martínez-Castruita, I. A., Sandoval-Ortega, M. H., ArellanoDelgado, M., \& Martínez-Calderón, V. M. (2021). Infestación por Cladocolea loniceroides y sus potenciales aves dispersoras de semillas en un área verde urbana de la ciudad de Aguascalientes, México. Madera y Bosques, 27(1). e2712084. doi: 10.21829/myb.2021.2712084

Madera y Bosques por Instituto de Ecología, A.C. se distribuye bajo una Licencia Creative Commons Atribución-NoComercialCompartirlgual 4.0 Internacional. 